\title{
DeVelopment of ConCRETE PIPE MOLding MACHINE WITH TOPOLOGY OPTIMIZATION
}

\author{
HoNG SEOK PARK \& PRAKASH DAHAL
}

Abstract: In this research, a new machine for manufacturing SPC (Sulphur Polymer Concrete) pipe is designed by taking consideration of inner rotating core for mixing, compressing and giving final shape of product. Research is focused to optimize the topology of part for ensuring robust functionality. First, the concept of a new molding machine was designed by applying TRIZ principles. Among the various concepts, one of the concepts was selected using scoring techniques, where concepts are presented and compared with varieties of evaluating criteria. Topology optimization with density method has applied to design inner rotating core part for mass reduction and thereby optimum utilization of design space. Objective is focused to minimize the developed topology by maximizing stiffness. Optimized finite element topology is generated as CAD model and response charts of different design variables are generated. CATIA, OptiStruct and ANSYS are tools used for concept design, optimization of topology. To the end optimal topology is compared with the initial designed part in terms of weight and displacement and is concluded that topology optimized model maximizes overall stiffness resulting in better product design.

Key words: Sulfur polymer concrete, Molding machine, Product design, Topology optimization
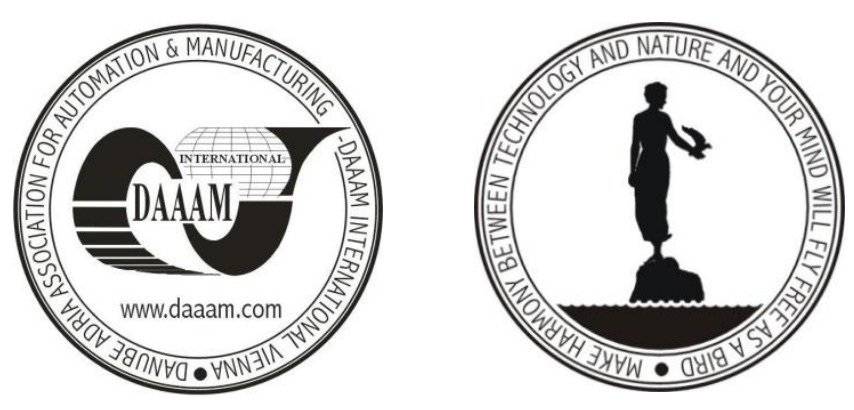

Authors' data: Univ.Prof. Dr.-Ing. Park, H[ong] S[eok]*; Graduate Researcher Dahal, P[rakash $]^{* *}, *$ Department of Mechanical Engineering, University of Ulsan, Daehak-ro 93, Nam-gu, Ulsan, Republic of Korea, **Department of Mechanical Engineering, University of Ulsan, Daehak-ro 93, Nam-gu, Ulsan, Republic of Korea, phosk@ulsan.ac.kr, prakashdahal@gmail.com

This Publication has to be referred as: Park, H[ong] S[eok] \& Dahal, $\mathrm{P}$ [rakash] (2013) Development of Concrete Pipe Molding Machine with Topology Optimization, Chapter 45 in DAAAM International Scientific Book 2013, pp. 751766, B. Katalinic \& Z. Tekic (Eds.), Published by DAAAM International, ISBN 9783-901509-94-0, ISSN 1726-9687, Vienna, Austria

DOI: $10.2507 /$ daaam.scibook.2013.45 
Park, H. S. \& Dahal, P.: Development of Concrete Pipe Molding Machine with Top...

\section{Introduction}

SPC is a new emerging material which is a mixture of sulfur, binder and variety of aggregates. Sulfur concrete is manufactured by allowing mixtures of the aggregates and molten sulfur to cool, whereupon the mixture solidifies to give products of hardness comparable to concrete. This concrete has unique properties including fast setting characteristics, high ultimate strength, low permeability, and resistance to acids and saline solutions. Although Portland cement concrete is widely used from the past but the strength deterioration is rapid due to the reaction of various chemical, mechanical and biological processes. Owing to this fact, sulfur concrete is rapidly increasing its demand due to its vast range of advantages. Sewer pipe made of sulfur concrete is inexpensive during construction, and after manufacturing it will provide better sewer systems and conduit extremely durable in most of the environments. Owing to this fact, this research is focused to develop the molding machine which will be able to manufacture the SPC sewage pipes. From the various experiments, it was found that there are several factors which are used to manufacture all concrete pipes as: strength at early age, surface texture and density, permeability, sulfate resistance, and consistency. Superior strength is generated due to fast curing time of material property whereas density and consistency of product will be obtained from manufacturing process. To obtain denser and consistent product, the die of molding machine is made to perform multi functions to provide compressibility and removing air voids during the manufacturing phase.

Corrosion of concrete sewer pipe is an existing problem that faces sewer systems all over the world. Sewer pipes are attacked by sulfuric acid that is derived from the biogenic activity or by direct oxidation of hydrogen sulfide from sewerage. Corrosion in sewage pipes leads to costly replacement of concrete structures therefore if the sewer pipes manufacturing process are not efficient and quality of pipe will not perform good function for a long period. The pipe manufacturing machines popular in now days are mostly horizontal spun type. Although concrete pipe manufacturing processes have changed throughout the years, each process ensured a durable product. Concrete pipe that is manufactured today is dense, strong and durable. As the demand for concrete pipe continued to grow, so did the need to increase output and productivity with uniformity of concrete particle distribution. Equipment manufacturers are interested to produce high quality pipe with faster rate. (OCPA ) Therefore new material with new manufacturing method is necessary to develop to cope of the requirement of pipe manufacturing company.

\section{Problem Description}

There are a lot of technologies to produce pipes of different shape and size including spun technology, center core vibration, and packer head etc. Until now spun technology is dominating in most of the industries to produce the concrete pipe which works on the principle by incorporating mechanism of outer core rotation in 
horizontal centrifugal machine. This machine operates on high speed RPM where concrete is feed into the rotating mold. This manufacturing operation is time consuming and efficiency is very low, in addition physical strength of the product quality is not good and thickness is not uniformly distributed.

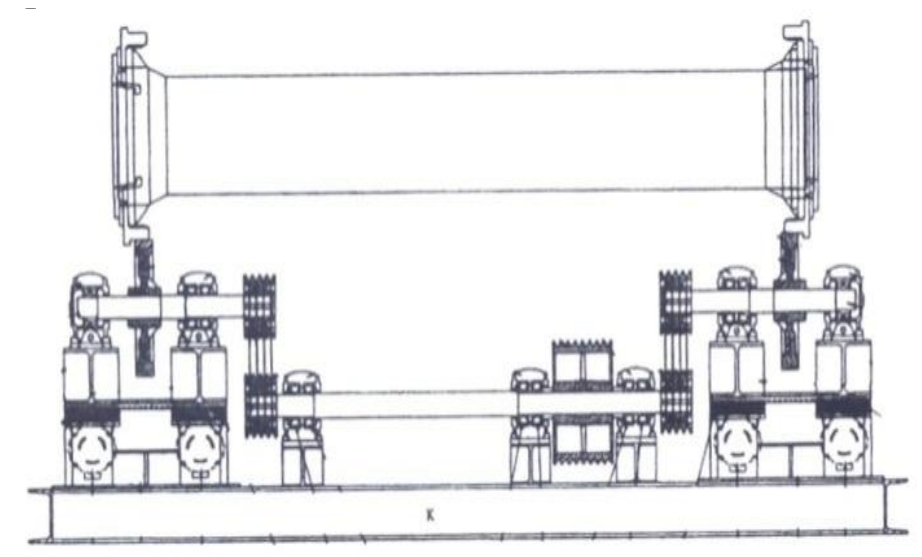

Fig. 1. Existing horizontal type spun machine

To improve the product quality following points must be considered for the new system:

1. Centrifugal force of conventional molding techniques must be replaced to develop new product of SPC.

2. Manufacturing facility must be more efficient which makes very less time to manufacture

In the current study, based on the existing horizontal centrifugal molding technology high frequency vibration is added and layout will be changed into vertical to ensure quality product. This process will improve the dimensional accuracy of the products and also enhances the manufacturing time. So, there is a great change in effectiveness of product when the manufacturing process changed to high frequency vibration with centrifugal force in vertical alignment. To complete this research task engineering design is done with systematic planning, following the scientific and practical ideas to meet the stated objectives.

\section{Development of Vertical Type Machine}

\subsection{Design Specification}

Design specification for the new machine was obtained from the existing pipe manufacturing company in South Korea. From the discussion with company a prototype machine will be developed which can manufacture the pipe of size shown in Fig 2. 
Park, H. S. \& Dahal, P.: Development of Concrete Pipe Molding Machine with Top...

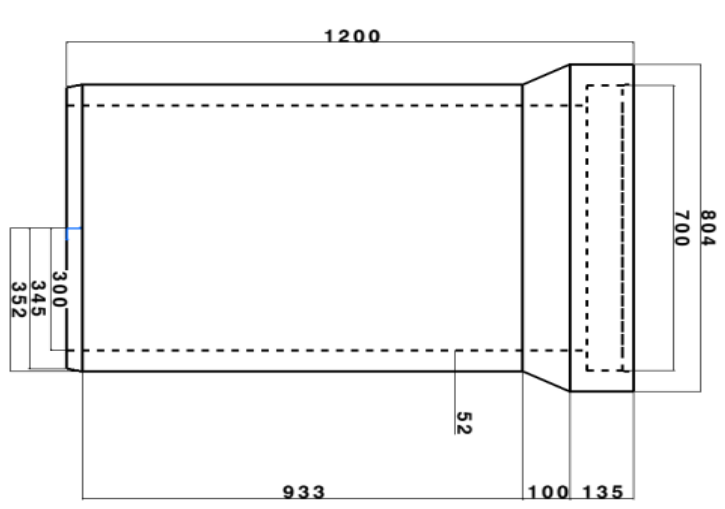

Fig. 2. Dimensions of pipe to be manufactured from prototype

Design requirements are the set of terminologies which are used to develop the final approach of a system. They can be classified into physical, structural, functional, cost requirements. Considering those requirements target prototype must fulfill the following requirements

1. Homogenous compaction of material

2. No cosmetic cracking at surface

3. No leakage

4. Able to produce product varieties

5. Can be easily operated

Therefore to have a chance of reliable durable product to be manufactured, system must be long lasting, requiring low maintenance, low cost and designed by sequential design process is preferred.

\section{Design Procedure}

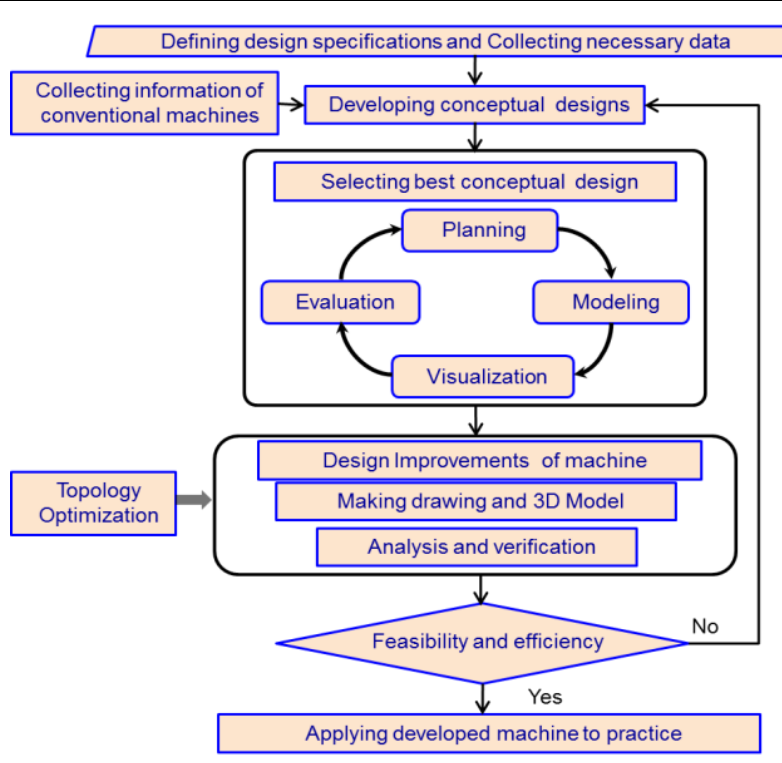

Fig. 3. Schematic of design process 
The practical design of molding machine starts with collection of necessary parameters, study of design specification. In this phase, several existing technologies and patents are reviewed to omit the patents and getting review of technology. Conceptual designs are created and realized by means of 3 dimensional CAD model to verify the functionalities of generated concepts. Concepts are designed in terms of performance, cost and ease of manufacturability. Generated conceptual designs are visualized to verify the kinematic behavior and production steps of SPC pipe. Similarly, all of the innovative concepts are evaluated by means of evaluation criteria for selection of the best design. Detail design was carried out from selected conceptual design which includes detail drawings of each components and 3D CAD model of machine. After making detail design, prototype will be built for validation in real environment.

\section{Conceptual Design}

In order to develop the high quality pipe, systematic procedure is followed, TRIZ methodology is employed to assist the way to get new models which in turn used to resolve the encountered problems of old machine.

\begin{tabular}{|c|c|l|}
\hline $\begin{array}{c}\text { Principle } \\
\text { No }\end{array}$ & $\begin{array}{c}\text { TRIZ } \\
\text { principles }\end{array}$ & \multicolumn{1}{|c|}{ Specific solution } \\
\hline 4 & Asymmetry & $\begin{array}{l}\text {-Stable design of product } \\
\text {-Flexibility in manufacturing operations }\end{array}$ \\
\hline 5 & Merging & $\begin{array}{l}\text {-Merging vibration and rotation functions to get better design and } \\
\text { improved functionality. }\end{array}$ \\
\hline 8 & Antiweight & $\begin{array}{l}\text {-Reduces energy consumption and less material } \\
\text { consumption. }\end{array}$ \\
\hline 14 & $\begin{array}{c}\text { Spheroidal- } \\
\text { ity }\end{array}$ & $\begin{array}{l}\text {-Make round smooth cylindrical shape } \\
\text {-Equal circulation of plastic concrete in all parts }\end{array}$ \\
\hline 18 & $\begin{array}{c}\text { Another } \\
\text { dimension }\end{array}$ & $\begin{array}{l}\text {-Traditional horizontal machine is replaced by vertical type to } \\
\text { reduce manufacturing space } \\
\text {-For shortening manufacturing time }\end{array}$ \\
\hline 20 & $\begin{array}{c}\text { Continuity of } \\
\text { viseful action }\end{array}$ & $\begin{array}{l}\text {-Mixing the concrete particles and removing unnecessary voids } \\
\text { within concrete, for making durable product }\end{array}$ \\
\hline 28 & $\begin{array}{c}\text { Mechanical } \\
\text { substitution }\end{array}$ & $\begin{array}{l}\text {-Replace able to produce varieties of products e.g circular pipe and } \\
\text { noise minimization. }\end{array}$ \\
\hline 29 & $\begin{array}{c}\text { Pneumatics } \\
\text { and } \\
\text { hydraulics }\end{array}$ & $\begin{array}{l}\text {-Use hydraulic or pneumatic damper for vibration table for Stable } \\
\text { operation }\end{array}$ \\
\hline
\end{tabular}

Tab. 1. Changing TRIZ principles to specific solution 
Park, H. S. \& Dahal, P.: Development of Concrete Pipe Molding Machine with Top...

TRIZ is a powerful methodology which can provide the conceptual solutions. TRIZ can offer competitive innovation ideas. (SOUCHKOV, 1988) This approach is based on the four steps: Identifying problems, Formulate the problem, the Prism of TRIZ, Search for Previously Well-Solved Problem, Look for Analogous Solutions and Adapt to Solution. According to this principle, technical uncertainties during the design are eliminated and focus on the target objective is considered. Product's appearances as well as aesthetics are also considered in first stage of conceptual phase. Concept design is aimed to give number of reliable models that satisfies the requirements. Conceptual model generation is based on the four steps of TRIZ design parameters which are focused in manufacturability of machine, durability, reliability and shape of product (Serban et al., 1988). Number of patents and TRIZ technology is studied to avoid the patents and to generate robust conceptual design. Based on the inventive principles of TRIZ, specific solutions are established for generating efficient machine (Serban et al., 1988).

By considering TRIZ principles concepts are generated via CAD model where each machine satisfies the design requirement.

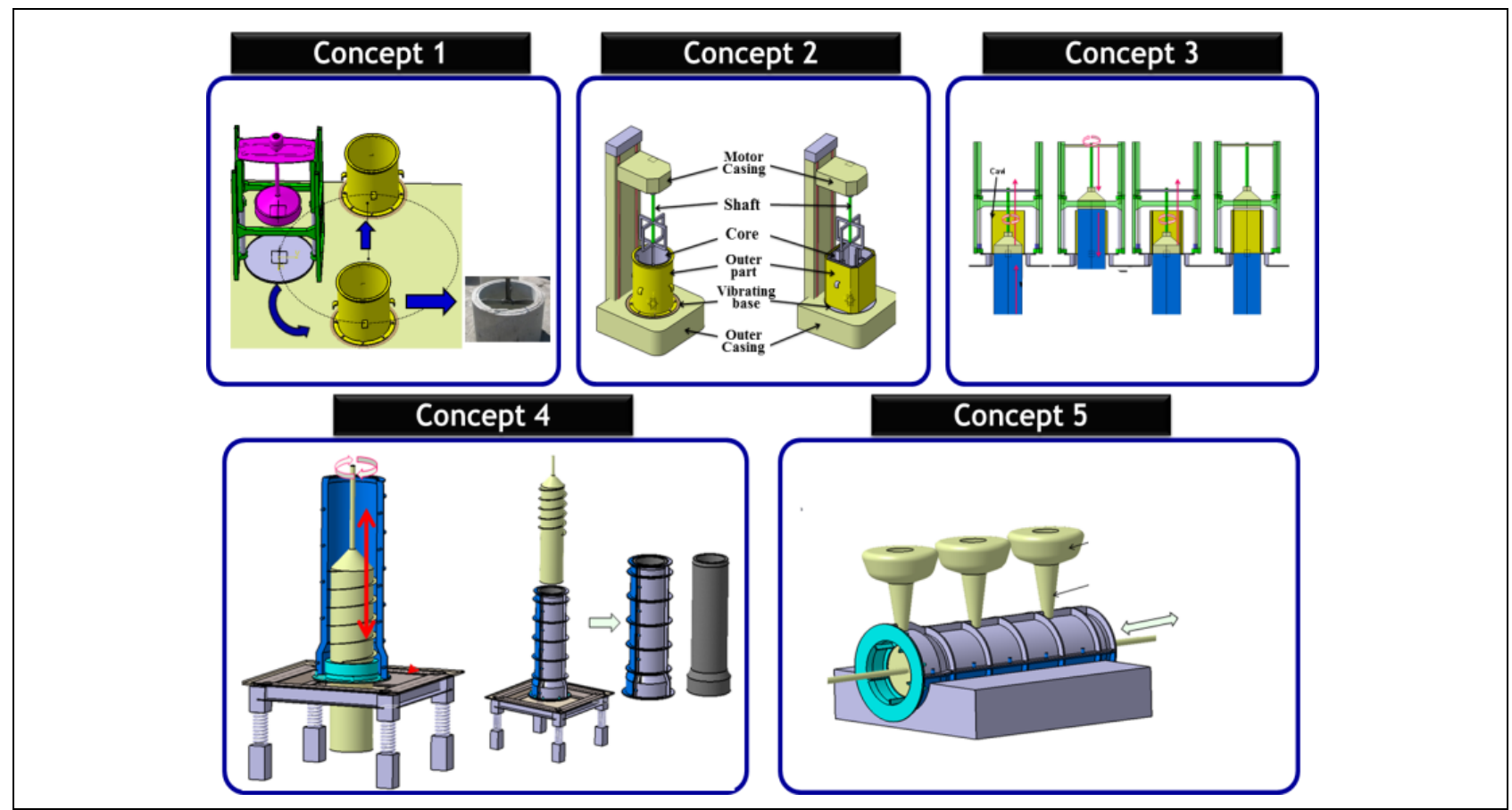

Fig. 4. Conceptual CAD models of SPC pipe molding machine

Concept 1 shows the model of machine having vibration base to give the vibration energy on the freshly inserted concrete. Vibration table is equipped with motor which is able to change the desired amplitudes, frequencies, and vibration during change in pipe size. Therefore, it is important to keep the concrete mixture in constant proportion regardless of the pipe sizes. Rotation is carried by the electric motors mounted at the top to provide more compaction and shaping of concrete. Inner die system is movable in up down motion during shaping of pipe. 
Concept 2: This unique concept is used to make circular as well as rectangular pipes. The positioning of the pipe is vertical mount system where the vibration is given from the base which is alike to the vibration as mentioned in concept 1 . This machine is able to provide not only vibration but also rotation. Outer die is fixed in the vibration table and inner die is movable which can move by means of rotation of shaft coupled with electric motor mounted at the top. In the inner die there are slots to couple the shaft with inner die so that when the shaft rotates the torque is transmitted to the inner die. So the product is speculated to perform better than existing products till now.

Concept 3: This model looks similar as model1 but the difference is inner core is equipped with vibration motor inside. Inner part consisting of two parts can be assembled and disassembled easily in the different steps of manufacturing as shown in Fig 4. During the first two steps the inner part generates vibration to remove air voids and compaction by rotation; whereas in the last two steps vibration is stopped and only small inner die portion is be rotated to give the smooth surface finish to the inner part. The smooth surface finish is given at last step to ensure low water permeability from the inner part of sewage pipe. The motion of inner core is provided by hydraulic mechanism whereas the rotation of inner part is provided by means of electric motor.

Concept 4: The vibration system for the concrete pipe making machine utilizes a precisely manufactured inner core that contains spiral shaped screw to generate pressure in the concrete of the inner mold. This setup consists of vibration table where preferably arranged electric vibrators are mounted at the frame of table. Vibration table is designed in the way that different size of pipe can be manufactured in the same table. To manufacture different sized pipe, only the changeable part is the upper plate of vibration table. The outer die and spigot end is fixed on the table at the first step then inner part is moved to its position and concrete is fed into the inner zone. Core starts to move in upward direction by compacting concrete. The core part can be adjusted to slide up and down from the small clearance hole at the center of table. The novel benefit of this machine is that it has vibration, compaction and centrifugal rotation mechanism by means of vibratory table and screw core respectively. The uniform distribution of vibration allows the concrete consolidation in the optimum manner and distribution of particles will be uniform.

Concept 5: This machine serves to cast pipes of concrete in horizontal pattern. Machine includes inner mold part, outer mold part. The inner core is displaced in horizontal axis after the production of pipe and outer die, finished pipe along with spigot end are taken to next place for storage. After the solidification of concrete, outer cover is taken to another place and finished pipe is taken out from die. Manufacturing steps of this embodiment is concrete is fed from the upper part of the die where there are holes to inject the pressurized concrete. Several holes are made to reduce the manufacturing time and for uniformity of concrete flow throughout the pipe cavity. Tiny air holes are also made at the upper part to remove the trapped air inside the cavity. 
Park, H. S. \& Dahal, P.: Development of Concrete Pipe Molding Machine with Top...

\section{Concept Selection}

Concept selection is the activity in which the generated concepts are analyzed and sequentially eliminated to identify the most promising concepts. A reference design is existing machine which is considered as the datum. Selection criteria are generated based on design requirements, which includes the customers' requirements and designers' intentions. For purpose of concept selection, the weight of the criteria can be determined subjectively by team consensus or through a designer's rational intent. A percentage is normally used to assign the weight.

\begin{tabular}{|c|c|c|c|c|c|c|c|c|c|c|c|}
\hline \multirow[b]{3}{*}{ Selection Criteria } & \multirow[b]{3}{*}{ Weignt } & & & & \multicolumn{3}{|c|}{ Concepts } & & & & \\
\hline & & \multicolumn{2}{|c|}{ Concept 1} & \multicolumn{2}{|c|}{ Concept 2} & \multicolumn{2}{|c|}{ Concept 3} & \multicolumn{2}{|c|}{ Concept 4} & \multicolumn{2}{|c|}{ Concept 5} \\
\hline & & Rating & weighted score & Rating & weighted score & Rating & weighted score & Rating & weighted score & Rating & weighted score \\
\hline Product variety & 8 & 2 & 16 & 5 & 40 & 3 & 24 & 5 & 40 & 3 & 24 \\
\hline No peneteration of liquids & 9 & 4 & 36 & 4 & 36 & 4 & 36 & 4 & 36 & 4 & 36 \\
\hline Ease in manufacturing & 6 & 3 & 18 & 4 & 24 & 3 & 18 & 3 & 18 & 5 & 30 \\
\hline Uniform density product & 7 & 3 & 21 & 3 & 21 & 4 & 28 & 5 & 35 & 4 & 28 \\
\hline Ease of Handling & 6 & 4 & 24 & 3 & 18 & 3 & 18 & 3 & 18 & 5 & 30 \\
\hline \multirow[t]{3}{*}{ Durable product } & 9 & 3 & 27 & 3 & 27 & 3 & 27 & 5 & 45 & 4 & 36 \\
\hline & & & 142 & & 166 & & 151 & & 192 & & 184 \\
\hline & Continue? & & No & & No & & No & & Yes & & No \\
\hline
\end{tabular}

Tab. 2. Concept scoring table for identification of best concept

Design concepts are rated through a comparison to the datum design. Representative ratings are valued from 1 to 5, indicating from much worse to much better designs than the reference. (Xiao et al) Once all the concepts are rated, a total score for each design is calculated using this formula. $\quad \mathrm{Sj}=\Sigma 1 \mathrm{n} \llbracket \mathrm{rij}$ wi $\rrbracket$ where, $\mathrm{n}$ is the number of selection criteria, rij is the rating of concept $\mathrm{j}$ for ith criterion, and wi is the rating of the ith criteria. The overall performances of the alternative designs are ranked based on their total score. The relative strengths and weaknesses of alternative concepts can be identified by investigating the concept scoring matrix which can be seen in table 2 . The concept scoring method can help designers to identify the strengths and weaknesses of alternative designs; and, by combining of the advantages of different designs, a designer can achieve an improved design to fulfill functional requirements. This technique is efficient, because when functional requirements are changed, only the weighting information needs to be adjusted to reflect the change; and, most of the scoring information can still be used. (Xiao et al) Best concept is selected and design improvement of the selected concept is done to improve the functionality of concept after concept scoring. Design improvement includes making machine components which are able to perform mechanical functionality when machine is functional. It can be tested by means of analysis of mechanisms, linkages, collision between the parts and realization of production process. Comparison is done in machines that can make different product varieties, ease in manufacturing, uniform density and durable product weight is given for each criterias; and by using the selection formula concept 4 is applied for further improvement. 


\section{System Improvement and Realization}

The target specifications set earlier in the process are revisited after a concept has been selected and tested. At this point, the whole system is reanalyzed with mechanical behavior for the realization of product concept for practicing to reality. Drive system of motor and linkages are fixed. Size of system is fixed to make the prototype pipe shown in Fig 5. Vibration table, inner die, outer die and support structures are made in CAD environment to realize the system workable for production of prototype pipe.

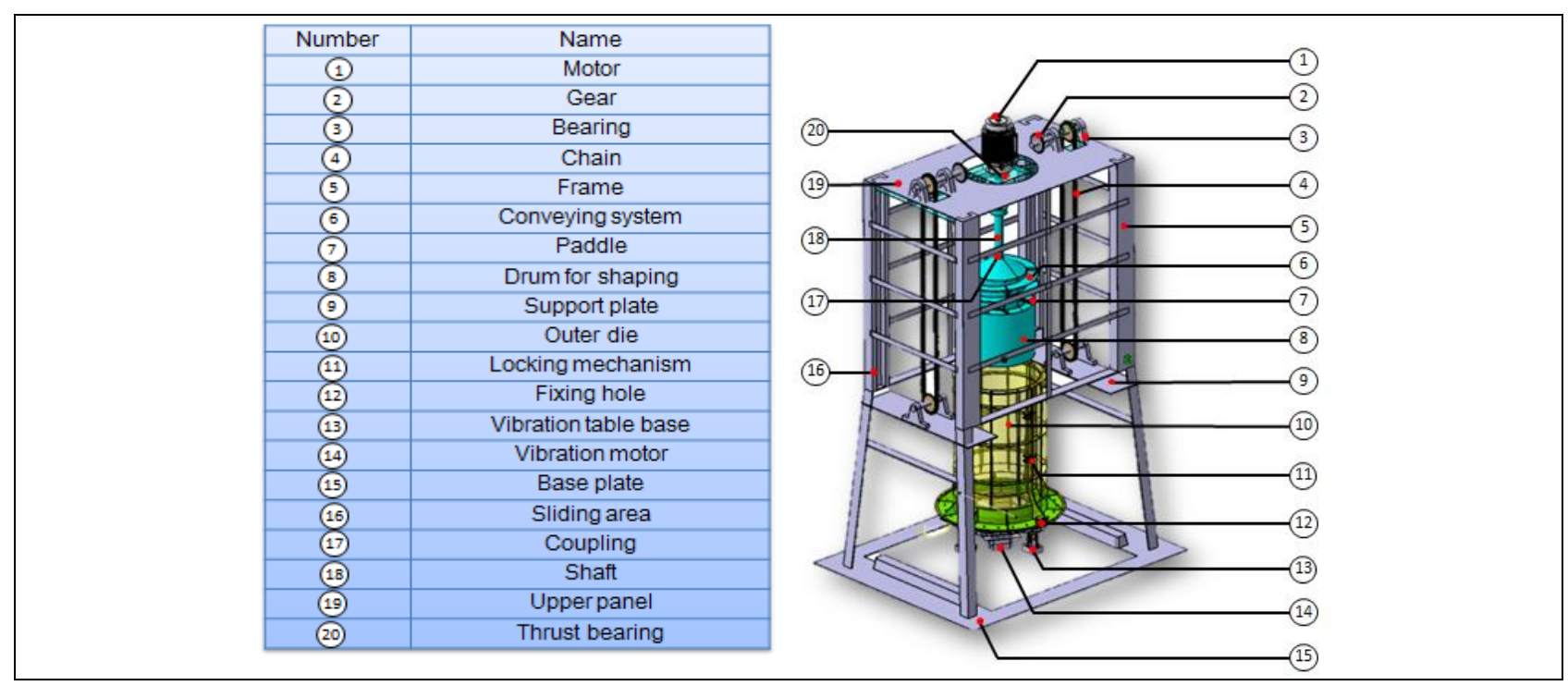

Fig. 5. Design improvement of concept system

\section{Topology Optimization of Inner Die}

\subsection{Introduction}

Ribs of inner die are used as a component for this study. Inner die being main component for rotation driven by rotation powered motor; therefore weight of inner die should be put as minimum as possible. Weight reduction will give substantial impact to fuel efficiency, efforts to reduce emissions and therefore, save environment. Weight can be reduced through several types of technological improvements, such as advances in materials, design and analysis methods, fabrication processes and optimization techniques, etc. These weight reducing strategies in industrial field can be achieved by means of different tools by performing optimization procedure. (Zurofi, 2004 1). Components must be designed to meet the requirements for strength and safety. Optimization methods were developed to have lighter, less cost and may have better strength too. There are four disciplines for optimization process in structural optimization strategy topology optimization, shape optimization, size optimization and topography. (Kojima, 2000) This optimization research aims to contribute to the development of structural design and weight reduction of inner die using topology optimization by the density method. Optimization process for this 
Park, H. S. \& Dahal, P.: Development of Concrete Pipe Molding Machine with Top...

work was conducted using OptiStruct solver in order to reduce the weight of the existing component.

\subsection{Methodology}

Optimization process used in current research is followed by several design steps firstly by making design domain of CAD model in CATIA. Next, Hyper mesh was used to import model for finite element modeling where loads and constraints are applied. Furthermore, Hyper view and Hyper graph were used to display and plot the data for results interpretation. Results of optimization runs performed with individual loads applied separately help to identify preferred load path with respect to the type of load applied.(Forcier \& Joncas, 2010,(Hsu, 2005) Finite element analysis is used in first stage before performing optimization to analyze the initially generated design. Optimization is performed and generated optimal model is further compared with initial model for verification of new design.

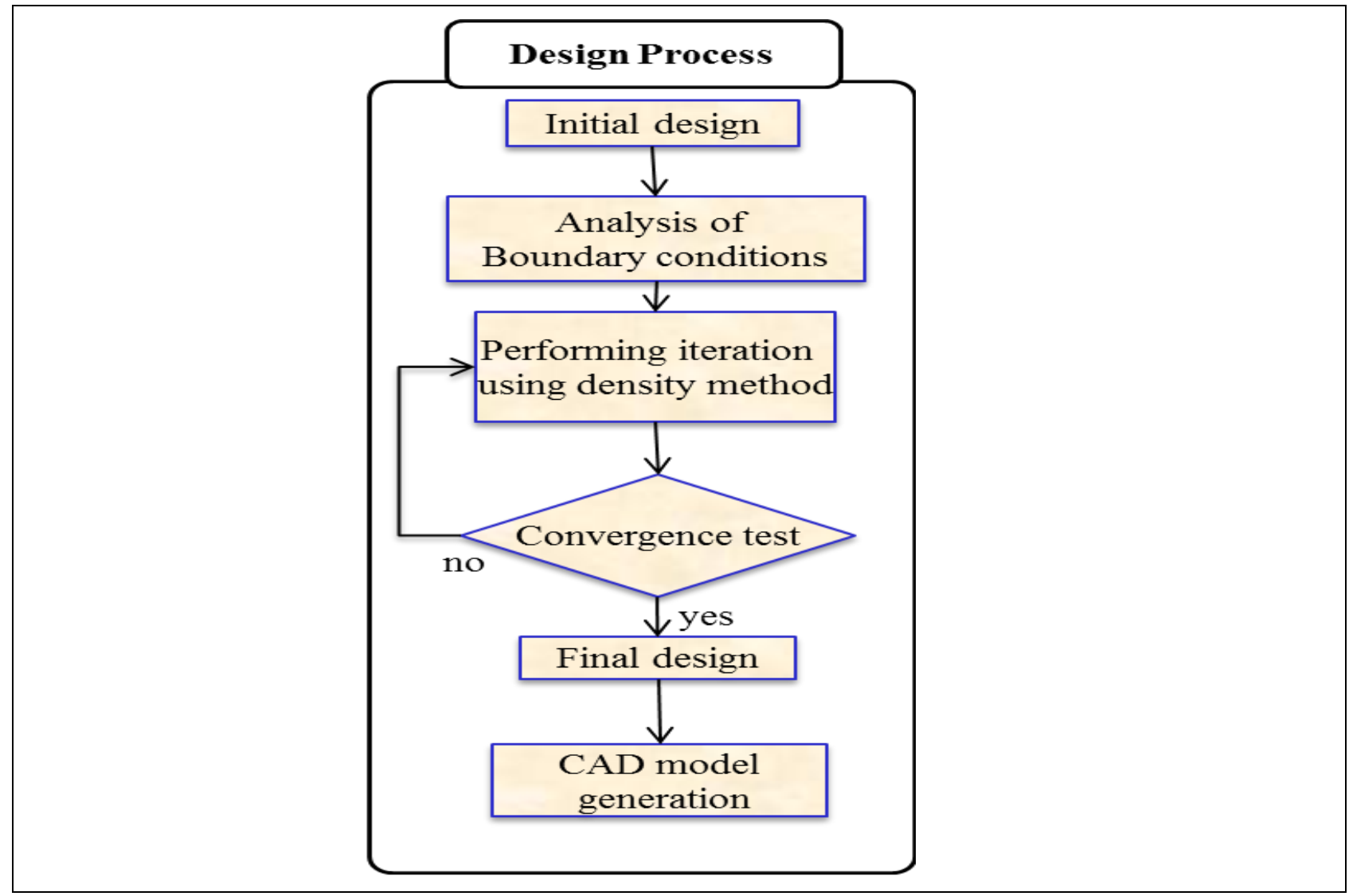

Fig. 6. Optimization procedure

\subsection{Optimization Problem}

For selecting inner die to generate optimal shape rib, design domain was considered. Keeping constant thickness of $5 \mathrm{~mm}$ from traditional rib, design domain is created as shown in Fig 7. Design domain is one portion of rib model among the six ribs from the whole inner die rib structure. Feasible optimal design topology is created by satisfying the optimization function. General optimization problem for topology optimization can be derived by 
Minimize $f(x)$

Subject to $(x)-\quad 0 j=1,2, \ldots . m$ $\mathrm{i}=1,2, \ldots . \mathrm{n}$

Where, $f(x)$ represents the objective function, $(x)$ and represents the $j$-th constraint response and its upper bound respectively. $\mathrm{M}$ is number of constraint. is normalized material density of $\mathrm{i}$-th element. Lower and upper bound of ith elements are between 0 and 1 . To enforce the design to be close to a $0 / 1$ solution, a penalty is introduced to introduce intermediate densities. For the SIMP approach the penalization is achieved by the following power law:

$\mathrm{Ei}=(\mathrm{xi}) \mathrm{p} \mathrm{E} 0$

Where, and are actual and original densities. Ei and E0 are actual young's modulus and original young's modulus; $p$ is penalization power. For practical applications it is important to support a variety of common analysis responses. In this research OptiStruct is used to get wide range responses for topology optimization including compliance and displacement response analysis. (Bendsoe \& Sigmund, 2004), (Altair OptiStruct) The optimization problem in this research is

Minimize Compliance $(\mathrm{C})=$ FTU

Subject to $\mathrm{V}=\mathrm{f} . \mathrm{VO}=$ eve

stress $2.5 \mathrm{e} 8 \mathrm{~N} / \mathrm{m} 2$ and Displacement $0.0357 \mathrm{~mm}$

where, the values are obtained from the material yield stress and results from the FE analysis. Finite element analysis is performed in consideration of loading condition during manufacturing process of SPC pipe. During the analysis maximum loading process is considered for worst case scenario.

Forces acting on the inner core are due to frictional force during the up down movement of core, centrifugal effect during rotation, pressure loads in concrete walls, drag force due to concrete during vertical movement of core in $\mathrm{Z}$ direction and frictional force during rotation. These forces are summarized as, FR1, FR2, FD3, FD4 where FR1 = sum of Pressure force at area 1 due to load of concrete + fricitional force at area 1 during rotation. FR2 is also same as FR1 however area is different.

FD3 and FD4 are drag force during inner die upward movement given by FD = $0.5 \mathrm{Cd} \rho \mathrm{A} \mathrm{V} 2$ Where, $\rho=$ Density of the fluid, $\mathrm{V}=$ speed of the object relative to the fluid, $\mathrm{Cd}=$ Drag coeff, $\mathrm{A}=$ Reference area. In the above expressions 1, 2, 3 and 4 are areas that are determined with respect to geometry of the consecutive areas (Fig.7). In this research force acting on the particle and particle interaction is not considered because target is to reduce the material in the inner die.

To perform optimization the structure of geometry is necessary to be minimized for reducing iteration time. Therefore one part of rib design domain was 
Park, H. S. \& Dahal, P.: Development of Concrete Pipe Molding Machine with Top...

considered. Upper part of design domain is connected with shaft therefore is fixed during optimization.

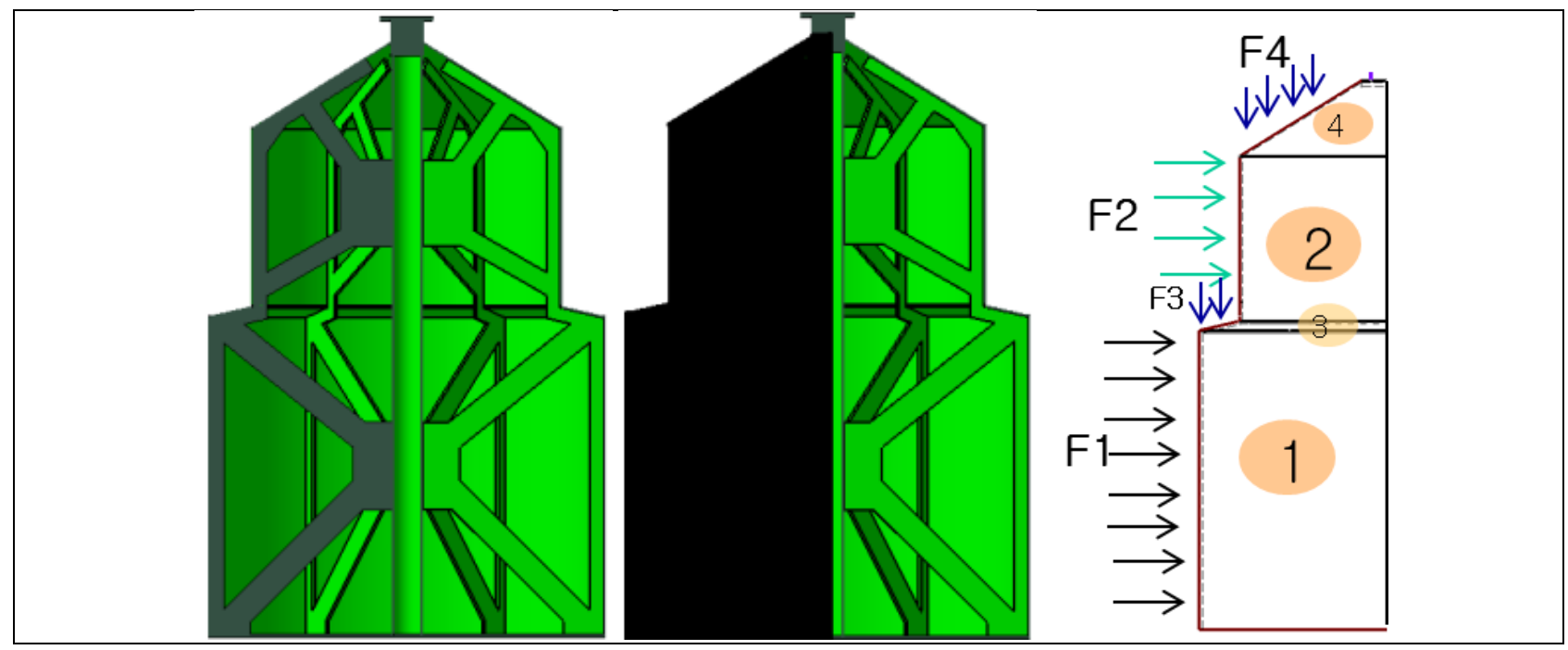

Fig. 7. Inner die design space considering symmetry

The CAD geometry of the traditional joint was generated by removing features such as fillets and other excessive details to facilitate meshing. From this geometry a design space was created. This design space was obtained by expanding the existing traditional geometry to create a very simplified envelope within which the final design must reside. This design envelope is determined by the maximum size of design domain.

The first stage in pre-processing was the definition of materials and element properties in HyperMesh. Once these were created with the appropriate qualities the model was meshed. A 3D tetramesh was created for the rib design space. The last input parameter required before implementation of the topology optimization study is the material identification. In the case of the inner core, the material was specified as steel; as a result, the material was defined as linear isotropic (MAT1) and the values for Young's modulus and Poisson's ratio were entered. These values used for the study were $2.1 \mathrm{e} 5(\mathrm{~N} / \mathrm{mm} 2)$ and 0.3 respectively.

The number of design variables is generally large in topology optimization; we need to obtain the design sensitivity with respect to each design variable to accurately and quickly find a local minimum design. In this case, the objective function is compliance when volume is minimized and the design variable is the normalized density so that we can easily obtain the numerical design sensitivity as follows:

$$
\begin{aligned}
& \frac{\partial \varphi}{\partial \boldsymbol{\rho}_{\mathbf{i}}} \\
& =\frac{\partial\left(\mathbf{F}^{\mathbf{T}} \mathbf{U}\right)}{\partial \boldsymbol{\rho}_{\mathbf{i}}} \\
& =\frac{\partial}{\partial \boldsymbol{\rho}_{\mathbf{i}}}\left(\mathbf{U}^{\mathbf{T}} \mathbf{K} \mathbf{U}\right)
\end{aligned}
$$




$$
\begin{aligned}
& =\frac{\partial\left(\mathbf{U}^{\mathrm{T}} \mathbf{K}\right)}{\partial \boldsymbol{\rho}_{\mathbf{i}}} \mathbf{U}+\frac{\left(\mathbf{U}^{\mathrm{T}} \mathbf{K}\right) \partial \mathbf{U}}{\partial \boldsymbol{\rho}_{\mathbf{i}}} \\
& =\mathbf{U}^{\mathrm{T}} \frac{\partial \mathbf{K}}{\partial \boldsymbol{\rho}_{\mathbf{i}}} \mathbf{U}+\mathbf{K} \frac{\partial \mathbf{U}^{\mathrm{T}}}{\partial \boldsymbol{\rho}_{\mathbf{i}}} \mathbf{U}+\frac{\left(\mathbf{U}^{\mathrm{T}} \mathbf{K}\right) \partial \mathbf{U}}{\partial \boldsymbol{\rho}_{\mathbf{i}}} \\
& =\mathbf{U}^{\mathrm{T}} \frac{\partial \mathbf{K}}{\partial \boldsymbol{\rho}_{\mathbf{i}}} \mathbf{U}+\frac{(\mathbf{K U}) \partial \mathbf{U}^{\mathrm{T}}+\left(\mathbf{U}^{\mathrm{T}} \mathbf{K}\right) \partial \mathbf{U}}{\partial \boldsymbol{\rho}_{\mathbf{i}}} \\
& =\mathbf{U}^{\mathrm{T}} \frac{\partial \mathbf{K}}{\partial \boldsymbol{\rho}_{\mathbf{i}}} \mathbf{U}+\frac{\mathbf{K}\left(\mathbf{U} \partial \mathbf{U}^{\mathrm{T}}+\mathbf{U}^{\mathrm{T}} \partial \mathbf{U}\right)}{\partial \boldsymbol{\rho}_{\mathbf{i}}} \quad\left(\text { Here } \frac{\mathbf{U} \partial \mathbf{U}^{\mathrm{T}}}{\partial \boldsymbol{\rho}_{\mathbf{i}}}=\frac{\mathbf{U}^{\mathrm{T}} \partial \mathbf{U}}{\partial \boldsymbol{\rho}_{\mathbf{i}}}\right) \\
& =\mathbf{U}^{\mathrm{T}} \frac{\partial \mathbf{K}}{\partial \boldsymbol{\rho}_{\mathbf{i}}} \mathbf{U}+\mathbf{2} \mathbf{U}^{\mathrm{T}} \mathbf{K} \frac{\partial \mathbf{U}}{\partial \boldsymbol{\rho}_{\mathbf{i}}} \\
& =-\mathbf{U}^{\mathbf{T}} \frac{\partial \mathbf{K}}{\partial \boldsymbol{p}_{\mathbf{i}}} \mathbf{U}
\end{aligned}
$$

It can be written in another form as

$$
-u_{i}^{\top} \frac{\partial k_{i}}{\partial \rho_{i}} u_{i}
$$

Where ${ }^{\varphi}$ is compliance, $\mathrm{K}$ is the global stiffness matrix, ui is the ith element displacement vector, and ki is the ith element stiffness matrix. (Kim et al., 2012)

From the sensitivities formula we can check the convergence of the optimal topology as

$$
\left|\frac{\mathrm{C}_{\mathrm{K}+1}-\mathrm{C}_{\mathrm{k}}}{\mathrm{C}_{\mathrm{k}}}\right|<\epsilon\left|\frac{\max \left(\mathrm{x}_{\mathrm{K}+1}\right)-\max \left(\mathrm{x}_{\mathrm{k}}\right)}{\max \left(\mathrm{x}_{\mathrm{k}}\right)}\right|<\epsilon
$$

The relative difference of the design variable and objective can be used as the judgment condition of a convergent result. (Zuo et al., 2007) After the optimization has processed, model is adjusted for a series of steps until the convergence is reached. The resulting geometry proposal obtained from optimization process can be seen in Fig 8. The graph shows the convergence value in each level of iterations to determine the maximum allowable limit of volume contained in the material under boundary conditions. When the value tries to become stable for several iterations then the convergence criteria is considered as fulfilled and the target is accomplished.

The pattern of rib is completely different than the proposed design at the first phase. The convergence graph shows below the objective function as minimizing volume. In 31 iterations the volume is converged from the single design domain to feasible geometry in design space. Main objective is focused to minimize the geometry by considering stress and displacement as a constraint. By applying the entire prerequisites information convergence graph is obtained as follows. 
Park, H. S. \& Dahal, P.: Development of Concrete Pipe Molding Machine with Top...

Although the topology results appear reasonable, the design is not ready to hand over to the machine shop for fabrication. The results of the topology studies are merely rough geometric proposals, and some interpretation is required to create the final design. OptiStruct has the ability to export the topology results as an IGES file using an export feature so that the geometry can be opened and traced over in a CAD environment.

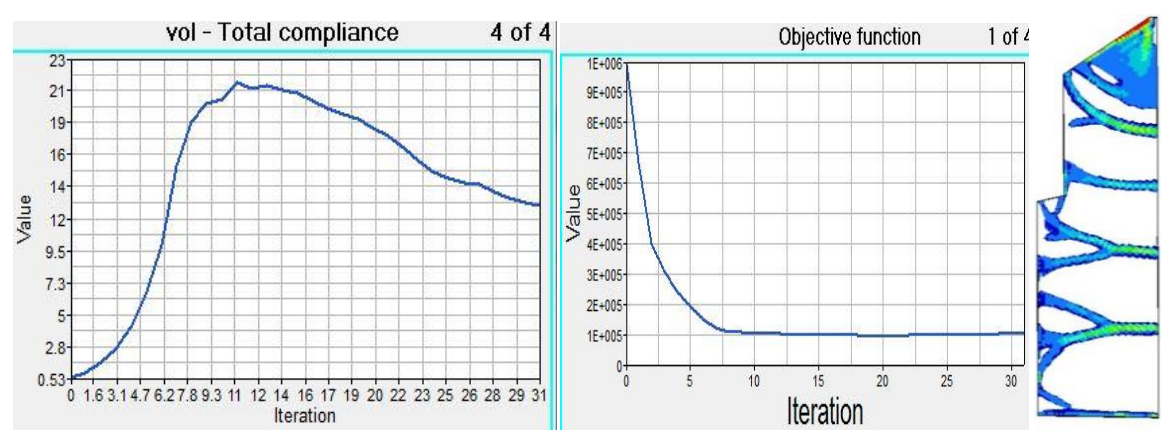

Fig. 8. Result of topology optimization

For the interpretation of the ribs of inner die, the optimized shape was created using CATIAV5. To capture the shape of the proposed design, sketch was done over the generated rough model for creation of the CAD model, and the interpretation can be seen in Fig 9.

The design retains the features proposed by OptiStruct to get better and efficient performance within the minimization of weight. The obtained design was rotated in circular pattern in six places to support the outer structure as same orientation with traditional design. The generated model after the optimization was found to be 9.5 percent lesser in weight than initial design.
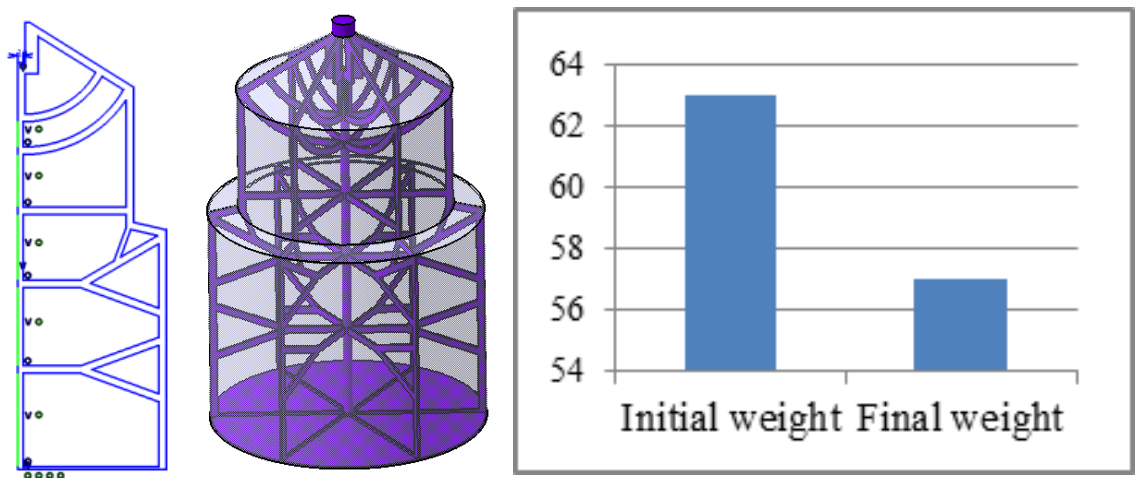

Fig. 9. CAD topology with weight comparison

Displacement analysis is also done to check the performance of structure. Initial displacement result is $0.035 \mathrm{~mm}$ during the full loading condition in whole structure whereas final displacement result is $0.034 \mathrm{~mm}$. The slight reduction in displacement 
verifies that although significant weight is reduced; displacement can also be minimized by proper distribution of material in given design space
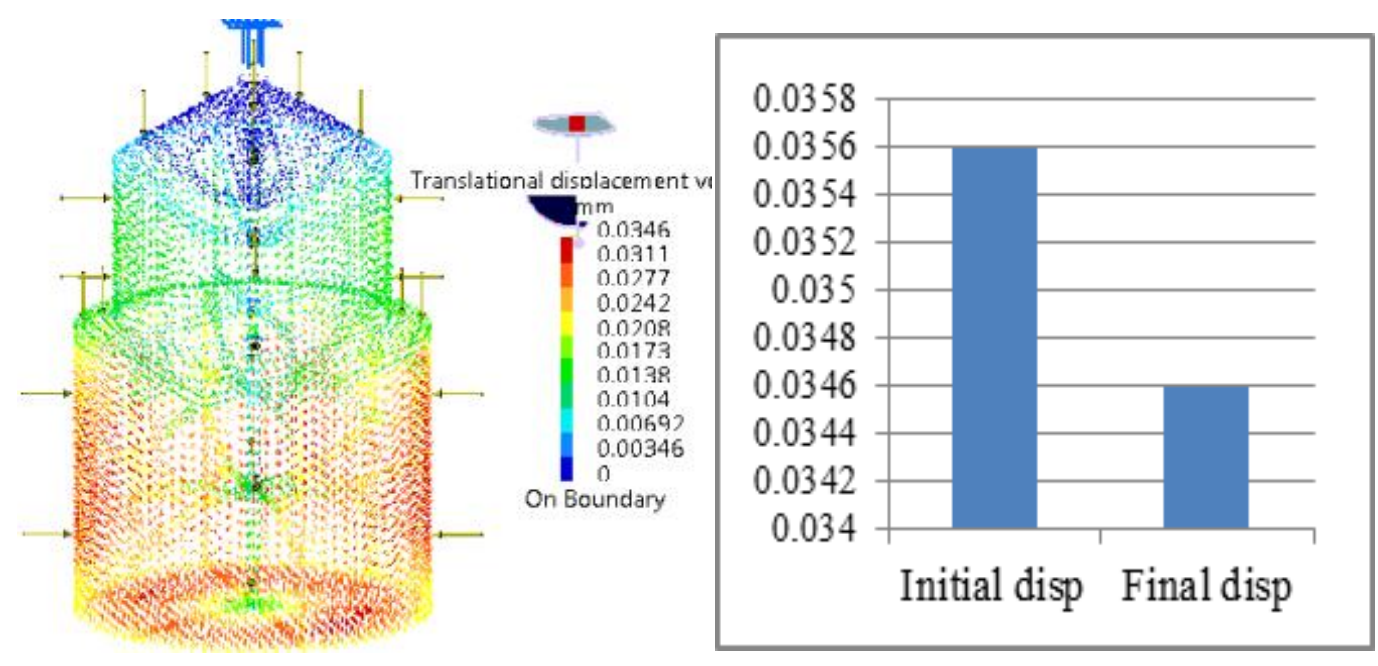

Fig. 10. Displacement analysis result comparison

This research introduced a procedure to apply topology optimization to rib designs for an inner core of newly designed molding machine. The rough configurations of ribs formed by solid elements are generated by topology optimization within a specified design space.

Post-processing work based on the generated rib topology and some engineering experience replaces the topology optimized structure to feasible structure for manufacturing. The effect of topology optimization is significant which does not only satisfies all constraints but also weights much less than the structure obtained by conventional methods as the weight is reduced to $6 \mathrm{Kg}$ from the research.

\section{Conclusion}

This research proposed a new concept molding machine capable of performing conveying, mixing, compacting and surface finishing during production of concrete pipes. Due to the new design concept and by following proper design process, the proposed design can be a milestone to satisfy the requirement of the pipe manufacturing companies. From the optimization strategy it is mentioned that basic shape from traditional design is changed to new shape. And the weight is less than compared with the original design.

Therefore, it can be verified that application of topology optimization in the conceptual design phase is reasonable for reducing weight. This is a moving structure therefore some amount of energy can be saved during manufacturing SPC pipe due to light weight design. The performed optimization technique is a robust technique which has seen small uses in particular research such as automotive and aerospace. 
Park, H. S. \& Dahal, P.: Development of Concrete Pipe Molding Machine with Top...

This research has shown that the same methods can be applied to manufacturing areas. By using this approach rib structure weight is reduced to 9.5 percent. The optimized design has been arrived numerically through the number of iterations but verification is needed as a future work. Although, the concept is generated but practical validation is needed in application for the real use.

\section{Acknowledgements}

This work was supported by Research Grant University of Ulsan 2012.

\section{References}

Adrian Xiao, Simon S. Park, Theo Freiheit; "A Comparison of Concept Selection in Concept Scoring and Axiomatic Design Methods", University of Calgary, Canada Altair OptiStruct, HyperWorks 10.0, User's manual. Altair Engineering, Inc D. Serban,E. Man, N. Ionescu and T. Roche; 2004 "A TRIZ Approach to Design for Environment", Product Engineering 89-100

Kojima,Y. 2000, "Mechanical CAE In Automotive Design", R\&D review of Toyota CRLD, Vol 35, No. 4

Kong-Tian Zuo, Li-Ping Chen, Yun-Qing Zhang. Jingzhou Yang, 2007, "Study of key algorithms in topology optimization" Int J Adv Manuf Technology 32: 787-796. Louis-Charles Forcier, Simon Joncas, 2010 "Development of a structural optimization strategy for the design of next generation large thermoplastic wind turbine blades" AIAA/ASME/ASCE/AHS/ASC Structures, Structural Dynamics, and Materials Conference, Orlando, FL, USA

M. P. Bendsøe, O. Sigmund. Topology Optimization - Theory, Methods and Applications. Springer, Berlin, Heidelberg, New York, 2nd edition, 2004

MING-HSIU HSU, YEH-LIANG HSU "Generalization of two- and threedimensional structural topology optimization. Taylor \& Francis, Vol. 37, No. 1, January 2005, 83-102

OCPA concrete pipe design manual, Ontario Concrete pipe association.

SOUCHKOV V., 1988, “TRIZ: A Systematic Approach to Conceptual Design”, Ideal Design Solution, Netherlands

Sun Yong Kim, Yong Kim and Chris K. Mechefske; 2012, "A New Efficient Convergence Criterion For Reducing Computational Expense In Topology Optimization: Reducible Design Variable Method"; International Journal For Numerical Methods In Engineering, 90:752-783

Zurofi M., 2004 "Manufacturing Process Effects on Fatigue Design and Optimization of Automotive Components- An Analytical and Experimental Study", The University of Toledo 